\title{
Elimination profile of triamcinolone hexacetonide and its metabolites in human urine and plasma after a single intra- articular administration
}

Sergi Coll ${ }^{1,2}$, Xavier Matabosch ${ }^{1}$, Jone Llorente-Onaindia ${ }^{3,4}$, Marcel·lí Carbó ${ }^{2}$, Clara Pérez-Mañá $^{5,6,7}$, Nuria Monfort ${ }^{1}$, Jordi Monfort ${ }^{3,4}$, Rosa Ventura ${ }^{1,2}$

(1) IMIM (Hospital del Mar Medical Research Institute), Catalonian Antidoping Laboratory, Barcelona, Spain

(2) Department of Experimental and Health Sciences, Universitat Pompeu Fabra, Barcelona, Spain

(3) IMIM (Hospital del Mar Medical Research Institute), Cell Research on Inflammation and Cartilage Group, Barcelona, Spain.

(4) Hospital del Mar de Barcelona, Rheumatology department, Barcelona, Spain

(5) IMIM (Hospital del Mar Medical Research Institute), Human Pharmacology and Clinical Neurosciences Research Group, Barcelona, Spain

(6) Department of Clinical Pharmacology, Hospital Universitari Germans Trias i Pujol (IGTP), Badalona, Spain

(7) Department of Pharmacology, Therapeutics and Toxicology, Autonomous University of Barcelona, Cerdanyola del Vallès, Spain

Corresponding author: Rosa Ventura Alemany

Doctor Aiguader 88, 08003 Barcelona, Spain

E-mail: rventura@imim.es

Phone: +0034-933160471

Fax: +0034-933160499 
Abstract

Triamcinolone hexacetonide (THA) is a synthetic glucocorticoid (GC) used by intraarticular (IA) administration. GCs are prohibited in sports competitions by systemic routes, and they are allowed by other routes considered of local action (IA administration, among others). The aim of the present work was to study the metabolic profile of THA in urine and plasma following IA administration.

Eight patients (4 males and 4 females) with knee osteoarthritis received an IA dose of THA (40 mg) in the knee joint. Spot urine and plasma samples were collected before injection and at different time periods up to day 23 and 10 post-administration, respectively. The samples were analysed by liquid chromatography-tandem mass spectrometry.

Neither THA nor specific THA metabolites were detected in urine. Triamcinolone acetonide (TA) and 6 $\beta$-hydroxy-triamcinolone acetonide were the main urinary metabolites. Maximum concentrations were obtained between 24 and $48 \mathrm{~h}$ after administration. Using the reporting level of $30 \mathrm{ng} / \mathrm{mL}$ to distinguish allowed from forbidden administrations of GCs, a large number of false adverse analytical findings would be reported up to day 4 . On the other hand, TA was detected in all plasma samples collected up to day 10 after administration. THA was also detected in plasma but at lower concentrations. The detection of plasma THA would be an unequivocal proof to demonstrate IA use of THA. A reversible decrease was observed in plasma concentrations of cortisol in some of the patients, indicating a systemic effect of the drug. 


\section{Introduction}

Triamcinolone hexacetonide (THA) is a synthetic glucocorticoid (GC) used in therapeutics by intra-articular (IA) administration (1). GCs are widely used in sports medicine for their anti-inflammatory and immunosuppressive properties in the treatment of different pathologies, including rheumatologic, hormonal, allergic, and other sports related injuries (2-6). In 1986, GCs were included in the list of forbidden substances in sports because of evidences of positive effects on exercise performance and health risks associated to their use due to the inhibition of the hypothalamic-pituitary-adrenal (HPA) axis (7-9). Since their inclusion, changes in their status have been produced in several revisions of the list with the aim of discriminating therapeutic use from misuse. In the current list of the World Anti-Doping Agency (WADA) (10), GCs are prohibited in sports competitions when administered by the so-called systemic routes (oral, intramuscular (IM), intravenous or rectal routes), and they are allowed for therapeutic purposes using other administration routes considered of local action (topical, intranasal, and IA administrations, among others).

In an attempt to discriminate allowed from forbidden administrations, WADA established a reporting level of $30 \mathrm{ng} / \mathrm{mL}$ for GCs. However, this general criterion is not suitable for all GCs for several reasons: the number of administration routes, differences in both metabolism and excretion rates as well as differences in pharmacological activity and therapeutic doses. As a consequence, a great research activity has been developed to find strategies to distinguish allowed from forbidden administration for some compounds of the group (11-21). For triamcinolone acetonide (TA), a drug related to THA, a previous study showed that a reporting level of $30 \mathrm{ng} / \mathrm{mL}$ was not able to detect forbidden IM administration. The use of a reporting level of $5 \mathrm{ng} / \mathrm{mL}$ was 
suggested to detect IM use as well as to distinguish IM from topical and intranasal administrations (19).

One of the main difficulties to find a criterion of discrimination between the currently allowed and forbidden administrations of GCs is that samples collected after allowed IA administration result in high concentrations in urine $(20,22-27)$, producing false adverse analytical findings (AAFs) according to the current rules. In addition, it has been shown that the metabolic profiles of several GCs after IA administration (betamethasone, methylprednisolone) are similar to IM administration (13, 20, 23, 24, 26). These findings illustrate the difficulties to distinguish between these administration pathways when evaluating the results of a single spot urine sample collected in a doping control.

On the other hand, some studies have shown a decrease in plasma concentrations of cortisol (CORT) after IA administration of some GCs, which indicates inhibition of the HPA axis, and therefore, a systemic effect (28-30). Apart from CORT plasma concentrations, some of the side effects observed after IA administration of GCs also suggest systemic effect $(31,32)$. However, some authors claim that these systemic effects are due to a wrong IA administration because of the difficulties to administer the GC inside the joint (33).

As far as we know, no data on urinary metabolites of THA has been reported up to now. THA is an ester of TA (Figure 1) and its hydrolysis to TA by plasma esterases is expected to occur when at least part of the dose administered reaches the blood stream after IA injection. Therefore, the urinary metabolites described for TA are also expected for THA. TA and six metabolites have been reported as the main compounds present in urine after TA administration $(18,19)$ (Figure 1). However, the possibility of the occurrence of specific metabolites of THA has never been tested. The detection of these 
specific THA metabolites in urine would be an unequivocal proof of IA use of THA. Accordingly, the detection of THA in plasma samples would be also a proof of IA use of THA.

The objective of this work was to study the urinary metabolites of THA after IA administration of the compound, as well as their elimination profiles. Plasma concentrations of THA and TA were also measured to evaluate the possibility to use THA in plasma as marker of IA administration. Finally, the systemic effect of THA after IA administration was evaluated by measuring plasma concentrations of CORT. 
2. Materials and methods

\subsection{Clinical study}

A clinical study that involved IA administration of THA to Caucasian subjects, and collection of urine and blood samples, was performed. (Protocol code: IMIMFTCL/BIOSAMPLE). Ethical approval of the study was granted by the local Research Ethical Committee (Comitè Ètic d'Investigació Clínica CEIC-Parc de Salut Mar, Barcelona, Spain).

A total of 8 subjects were recruited (Table 1). Due to difficulties to obtain ethical approval to use IA administrations in healthy volunteers, participants of the study were patients with osteoarthritis of the knee recruited in the Rheumatology Department of the Hospital del Mar (Barcelona, Spain). All participants gave their written informed consent prior inclusion. Most of the patients had controlled concomitant pathologies and took medications on a regular basis.

A single dose of $40 \mathrm{mg}$ was injected to each patient in the knee joint $(2 \mathrm{~mL}$ of Hexatrione 2\%, Laboratories Pharmaceutiques DEXO, Saint-Cloud, France). To ensure correct IA administration, all injections were carried out by the same medical doctor with extensive experience in IA treatments.

Spot urine samples were collected just before administration, $12 \mathrm{~h}$ after administration, and first-morning spot urines were collected on days 1 to $10,12,14,16,18,21$, and 23 post-administration. Urine samples were collected in sterile containers. Preliminary measurements, including specific gravity $(\mathrm{SG})$ and $\mathrm{pH}$, were performed. $\mathrm{SG}$ was determined using an Atago UG- $\alpha$ Refractometer (Atago, Tokyo, Japan), and $\mathrm{pH}$ was measured by a Crison GLP 21 pH meter (Crison, Alella, Spain). Urine samples were stored at $-20^{\circ} \mathrm{C}$ until further analysis. 
Blood samples were drawn just before administration, 1 and $2 \mathrm{~h}$ after injection and on days 1 to 4 and 10 after administration. All blood samples were collected in the morning in order to reduce the effect of circadian rhythm in CORT measurements (34). Blood samples were collected with $\mathrm{K}_{2}$ EDTA tubes (BD Vacutainer® spray-coated $\mathrm{K}_{2}$ EDTA Tube) according to the WADA blood collection guidelines (35). Plasma was obtained by centrifugation (10 minutes, $3000 \mathrm{rpm}, 4^{\circ} \mathrm{C}$ ), and was immediately transferred to lowbinding tubes and stored at $-20^{\circ} \mathrm{C}$ until analysis.

\subsection{Chemicals and reagents}

Triamcinolone acetonide (TA), triamcinolone hexacetonide (THA), triamcinolone (T), cortisol (CORT), cortisol-9,11,12,12-d4 (CORT-d4) and ammonium formate were

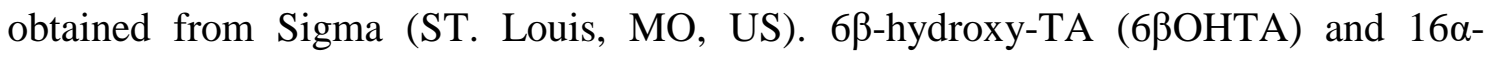
hydroxy-prednisolone-11,12,12,21,21-d5 (16aOHPRED-d5) were purchased from Toronto Research Chemicals (Toronto, Canada). Triamcinolone-6-d1 acetonide-d6 (TA-d7) was obtained from CDN Isotopes (Pointe-Claire, Canada). The $\beta$ glucuronidase preparation (type Escherichia coli K12) was purchased from Roche Diagnostics, GmbH (Manheim, Germany). Analytical grade disodium hydrogen phosphate, sodium hydrogen phosphate, potassium carbonate, ethyl acetate, acetonitrile and methanol (liquid chromatography gradient grade), and formic acid (liquid chromatrography-mass spectrometry grade) were obtained from Merck (Darmstadt, Germany). Milli-Q water was obtained using a Milli-Q purification system (Millipore Ibérica, Barcelona, Spain). Human blank plasma was provided by Hospital del Mar (Barcelona, Spain). 


\subsection{Sample preparation}

\subsubsection{Urine samples}

The analysis of urine samples was performed using an extraction protocol previously described by our group (19). Briefly, two internal standards (ISTDs) (40 ng of TA-d7 and $80 \mathrm{ng}$ of $16 \alpha \mathrm{OHPRED}-\mathrm{d} 5)$ were added to $5 \mathrm{~mL}$ of urine, followed by the addition of $1.5 \mathrm{~mL} 1 \mathrm{M}$ phosphate buffer $\mathrm{pH}$ 7. Then, enzymatic hydrolysis was performed by adding $50 \mu \mathrm{L}$ of $\beta$-glucuronidase enzyme from Escherichia coli and incubating for $1 \mathrm{~h}$ in a $55^{\circ} \mathrm{C}$ water bath. The mixture was alkalinized with $400 \mu \mathrm{L}$ of $25 \%$ potassium

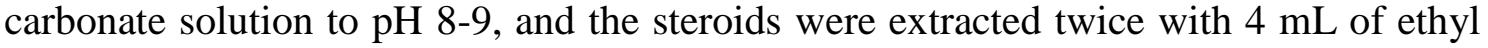
acetate. After centrifugation ( $5 \mathrm{~min}$ at $1400 \mathrm{~g}$ ), the organic layer was transferred into a new tube and evaporated to dryness under a nitrogen stream in a water bath at $40^{\circ} \mathrm{C}$. The residue was reconstituted into $100 \mu \mathrm{L}$ of a mixture of water to acetonitrile (3:1, $\mathrm{v} / \mathrm{v})$.

\subsubsection{Plasma samples}

For the analysis of plasma samples, two ISTDs $(50 \mu \mathrm{g}$ of CORT-d4 and $2.5 \mu \mathrm{g}$ of TAd7) were added to $1 \mathrm{~mL}$ of plasma, followed by the addition of $1 \mathrm{~mL}$ of acetonitrile in order to precipitate proteins. Then, steroids were extracted with $5 \mathrm{~mL}$ of tert-butylmethyl ether, and after centrifugation (10 $\mathrm{min}$ at $1400 \mathrm{~g})$, the organic layer was transferred into a new tube and evaporated to dryness under a nitrogen stream in a water bath at $40^{\circ} \mathrm{C}$. The residue was reconstituted into $150 \mu \mathrm{L}$ of a mixture of water to acetonitrile $(1: 1, \mathrm{v} / \mathrm{v})$. 
2.4 LC-MS/MS analyses

LC-MS/MS analyses were carried out using a triple quadrupole (Xevo TQ MS) mass spectrometer provided with an orthogonal Z-spray-electrosptray interface (Waters Associates, Mildford, MA, USA) coupled to an Acquity ultraperformance liquid chromatographic (UPLC) system (Waters Associates) for the chromatographic separation. Nitrogen and argon (both with purity $\geq 99.999 \%$ ) were used as desolvation gas and as collision gas, respectively. The desolvation gas flow was set to approximately $1200 \mathrm{~L} / \mathrm{h}$ and the cone gas flow to $50 \mathrm{~L} / \mathrm{h}$. The nitrogen desolvation temperature was set to $450^{\circ} \mathrm{C}$ and the source temperature to $120^{\circ} \mathrm{C}$. Capillary voltage of $3.5 \mathrm{kV}$ was used in positive ionization mode.

Liquid chromatographic separations were performed using an Acquity BEH C18 column (100 x $2.1 \mathrm{~mm}$ internal diameter, $1.7 \mu \mathrm{m}$ particle size) (Waters Associates) using a column temperature of $55^{\circ} \mathrm{C}$. The mobile phase solvents $\mathrm{A}$ and $\mathrm{B}$ were water and methanol, respectively, both with $1 \mathrm{mM}$ ammonium formate and formic acid $(0.01 \%)$. Two different liquid chromatographic methods were used. To analyse urine samples, the gradient used was already reported by our group to separate TA and its main metabolites (19). The percentage of organic solvent was linearly changed as follows: $0 \mathrm{~min}, 28 \% \mathrm{~B}$; $0.5 \mathrm{~min}, 28 \% \mathrm{~B} ; 6 \mathrm{~min}, 40 \% \mathrm{~B} ; 20 \mathrm{~min}, 44 \% \mathrm{~B} ; 20.5 \mathrm{~min}, 90 \%$ $\mathrm{B} ; 21 \mathrm{~min}, 90 \% \mathrm{~B} ; 21.1 \mathrm{~min}, 28 \% \mathrm{~B}$; and $23 \mathrm{~min}, 28 \% \mathrm{~B}$ at a flow rate of $0.3 \mathrm{~mL} / \mathrm{min}$. For the analysis of plasma samples, the percentage of organic solvent was linearly changed as follows: $0 \mathrm{~min}, 30 \% \mathrm{~B} ; 0.5 \mathrm{~min}, 30 \% \mathrm{~B} ; 3 \mathrm{~min}, 43 \% \mathrm{~B} ; 12.5 \mathrm{~min}, 48 \% \mathrm{~B}$; $13.5 \min , 65 \% \mathrm{~B} ; 16.5 \min 95 \% \mathrm{~B}, 16.6 \min 30 \% \mathrm{~B}$, and $18.5 \mathrm{~min}, 30 \% \mathrm{~B}$ at a flow rate of $0.3 \mathrm{~mL} / \mathrm{min}$. The injection volume in both chromatographic methods was $10 \mu \mathrm{L}$. 
For the study of THA metabolism, different mass spectrometric methods were used. Product ion scan analyses of THA were performed in positive and negative modes at different collision energies (CE) using direct infusion of the methanolic solution of the compound. Cone voltages $(\mathrm{CV})$ used in positive and negative modes were $25 \mathrm{~V}$ and 15 $\mathrm{V}$, respectively. Different methods were applied to detect THA metabolites in urine: open scan methods (Table 1S, Supplementary Information) and multiple reaction monitoring methods (MRM) using theoretical transitions of potential THA metabolites (Table 2S, Supplementary Information).

Urine and plasma samples from patients obtained in the clinical study were analysed using MRM methods described in Table 2 and 3, respectively. THA and TA and six metabolites previously described (Figure 1) $(18,19)$ were measured in urine samples (Table 2). In plasma samples, THA, TA and CORT were measured (Table 3). 
2.5 Validation of the method to analyze plasma samples

The validation of the method to quantify TA and metabolites in urine was presented in a previous study by our group (19). The analytical method to quantify concentrations of TA, THA and CORT in plasma samples was validated in the present work. The selectivity and specificity for the analysis of TA and THA was verified by analysis of 20 different blank of human plasma samples and checking for the absence of interfering substances at the retention times of the compounds.

For quantitative purposes, calibration samples at different concentration levels were prepared by adding the appropriate volume of stock solutions of the compounds to blank human plasma $(0.05,0.2,1,5,10$ and $20 \mathrm{ng} / \mathrm{mL}$ for TA and THA) or Milli-Q water $(2,5,10,20,50,100$ and $250 \mathrm{ng} / \mathrm{mL}$ for CORT). The peak ratios between the analyte and the corresponding ISTD were plotted against the concentration. Peak area ratios were subjected to a proportional weighted least-square regression analysis and calibration curves were not forced through the origin. TA-d7 was used as ISTD to quantify TA and THA, and CORT-d4 was used to quantify CORT.

Quality control (QC) samples at three concentration levels were prepared to evaluate the intra- and inter-assay precision and accuracy of the method. The QC samples were always analysed in triplicate. Accuracy is expressed as the relative error (\%) in the estimation of the concentration for the QC samples. Precision of the method is expressed as the relative standard deviation (\%) of the estimated concentrations.

Limits of detection (LOD) and quantitation (LOQ) were defined as 3.3 and 10 times the noise level, respectively. The standard deviation of the estimated concentration of the lowest calibration point for each analyte $(n=4)$ was used as a measure of noise. 
The extraction recovery was calculated by analysis of four replicates of a blank plasma samples spiked at three different concentrations of the compounds and four replicates of blank plasma samples to which the same concentrations of analytes were added after extraction of the blank matrix. The ratios of the peak areas between the analytes and the ISTD obtained from the extracted spiked samples were compared with the ratios obtained in the samples which analytes were added to extracted blank plasma samples (representing $100 \%$ of extraction recovery).

Ion suppression effect was studied by the analysis $(n=4)$ of four different blank plasma samples spiked with $1 \mathrm{ng} / \mathrm{mL}$ of TA, THA and TA-d7. The signal of the analytes and the ISTDs were compared with those obtained after the analysis of a water sample spiked with the compounds.

\subsection{Analysis of data}

Urinary concentrations of metabolites were corrected by the WADA SG-adjustment formula (36), where concentrations are adjusted to a reference value of 1.020 following the Levine-Fahy equation (37):

$$
C_{\mathrm{SG}}\left(\frac{n g}{m L}\right)=C\left(\frac{n g}{m L}\right) \times\left(\frac{1.020-1}{S G_{\text {sample }}-1}\right)
$$

Evaluation of gender differences in SG values was performed by t-student test. The level of significance was established at $\mathrm{p}<0.05$. Statistical analysis was performed using computer software SPSS 12.0 (SPSS Inc., Chicago, IL, USA). 


\section{Results}

\subsection{Detection of THA urinary metabolites}

First of all, the MS behaviour of THA was studied in order to find characteristic ionization and collision induced dissociation (CID) patterns that could be used to detect unknown THA metabolites in urine. In electrospray positive mode, THA was ionized at the conjugated 3-keto function forming the $[\mathrm{M}+\mathrm{H}]^{+}$ion and, in negative mode, forming the adduct $[\mathrm{M}+\mathrm{HCOO}]^{-}$, as described for other GCs $(12,14,15,18)$.

CID mass spectra of $[\mathrm{M}+\mathrm{H}]^{+}$and $[\mathrm{M}+\mathrm{HCOO}]^{-}$were obtained at different $\mathrm{CE}$ (Figure 2). In positive mode, the common fragmentation reported for GCs containing fluorine and acetonide groups (12) was obtained for the $[\mathrm{M}+\mathrm{H}]^{+}$of THA. Therefore, in the present study, the following ions were observed: ions formed by loss of $20 \mathrm{Da}(\mathrm{HF}), \mathrm{m} / \mathrm{z}$ 533>513; loss of $18 \mathrm{Da}\left(\mathrm{H}_{2} \mathrm{O}\right), \mathrm{m} / \mathrm{z} 513>495, \mathrm{~m} / \mathrm{z} 415>397, \mathrm{~m} / \mathrm{z} 357>339$ and $\mathrm{m} / \mathrm{z}$ 339>321; loss of $58 \mathrm{Da}$ (acetone), m/z 533>475, m/z 513>455 and m/z 415>357; and loss of $28 \mathrm{Da}(\mathrm{CO}), \mathrm{m} / \mathrm{z} 339>311$ and $\mathrm{m} / \mathrm{z} 321>293$. Ions due to the presence of the tertbutylacetyl moiety of THA were also observed: ions formed by loss of 118 Da (butylacetyl moiety+HF), m/z 533>415; loss of $136 \mathrm{Da}$ (butylacetyl moiety $+\mathrm{HF}+\mathrm{H}_{2} \mathrm{O}$ ), $\mathrm{m} / \mathrm{z}$ 533>397; and loss of $176 \mathrm{Da}$ (butylacetyl moiety $+\mathrm{HF}+\mathrm{H}_{2} \mathrm{O}+$ acetone), $\mathrm{m} / \mathrm{z}$ 533>357. Moreover, ions at m/z 121, 147 and 171 that are typical of the 1,4-diene GCs were observed at high $\mathrm{CE}(12,14,15,18)$. In negative mode, different product ions resulting from the loss of formic acid coming from $[\mathrm{M}+\mathrm{HCOO}]^{-}$were observed. A neutral loss of $220 \mathrm{Da}$, corresponding to the loss of $\mathrm{HF}, \mathrm{H}_{2} \mathrm{O}$, acetone and the tertbutylacetyl moiety, was observed $\mathrm{m} / \mathrm{z} 577>357$. An ion at $\mathrm{m} / \mathrm{z} 115$ corresponding to the 3,3-dimethyl butanoate group was present. In addition, the common fragmentation observed for other GCs containing fluorine and acetonide groups was also observed: 
ions formed by loss of $66 \mathrm{Da}(\mathrm{HCOOH}+\mathrm{HF}), \mathrm{m} / \mathrm{z} 577>511$; loss of $104 \mathrm{Da}$ (HCOOH+acetone), m/z 577>473; and loss of $20 \mathrm{Da}(\mathrm{HF}), \mathrm{m} / \mathrm{z}$ 357>337.

Based on the characteristic ionization and CID observed, two approaches were used to detect urinary THA metabolites: open scan methods (Table 1S, Supplementary Information), and MRM methods that included theoretical transitions for potential metabolites considering hydroxylations, oxidations and reductions of THA (Table 2S, Supplementary Information). Urine samples obtained after THA administration were analysed using these methods. Neither THA nor specific metabolites resulting from hydroxylations, oxidations and reductions of THA were detected in any of the samples (the LOD of THA in urine was $0.05 \mathrm{ng} / \mathrm{mL}$ ). The only metabolites detected were TA and those already described for TA $(18,19)$.

3.2 Analysis of urine samples obtained after THA administration Since only TA and its metabolites were detected in urines obtained after administration of THA, an already reported method validated for TA and its main metabolites (19) was used to analyse the urine samples of the patients recruited in the present study. TA, 6ßOHTA, T and four additional metabolites (M2 to M5) (Figure 1), were measured in all collected samples and the excretion profiles were evaluated. For metabolites M2 to M5, which were not available as standards, the excretion profiles were assessed using the relative signal of the compound to the ISTD. The sample preparation procedure applied allows for the detection of both free and glucoronoconjugated metabolites.

Concentrations of TA and 6 $\beta$ OHTA are presented in Figure 3 and Table 4, and concentrations of $\mathrm{T}$ together with the qualitatively determined metabolites (M2 to M5) are depicted in Figure 4. The main metabolite detected after IA administration of THA was $6 \beta$ OHTA, as reported for TA administrations (19). As mentioned above, THA was 
not detected in any of the samples analysed. Both TA and 6ßOHTA were detected up to

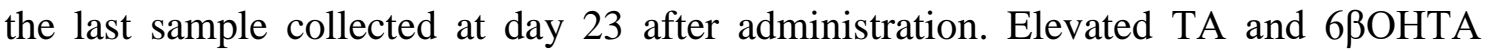
concentrations were obtained from day 1 to day 4 (Figure 3 and Table 4 ). In this time period, some metabolite concentrations were higher in males than in females, especially TA, M3, M4 and M5.

Three of the four female patients used medications containing diuretics, and this is probably the reason of the lower concentrations observed for females. In fact, male patients presented statistically higher SG values than female patients $(1.014 \pm 0.005$ and $1.012 \pm 0.005$ for males and females, respectively). Data corrected by SG are presented in Figures $1 \mathrm{~S}$ and $2 \mathrm{~S}$ (Supplementary Information). As can be seen, correction by SG did not reduce the gender differences for TA and metabolites M3, M4 and M5.

\subsection{Plasma samples}

\subsubsection{Method validation}

In contrast to the results obtained in urine, THA was detected in plasma samples after IA administration of the drug. An LC-MS/MS method was validated for the quantitation of THA, TA and CORT in plasma, and a summary of the results is presented in Table 3S (Supplementary Information). As can be seen, LOQ of TA, THA and CORT were 0.05, 0.05 and $2 \mathrm{ng} / \mathrm{mL}$, respectively. For all compounds, the assay was linear in the studied range of concentrations $\left(r^{2}>0.99\right)$. No significant interferences were observed at the retention times of the analytes and their ISTDs in the chromatograms of the corresponding ion transitions in the drug-free samples. Recoveries greater than $85 \%$ were obtained for all compounds, and no effect of the concentration on the extraction 
recoveries was observed. Accuracy and precision results showed that the assay is consistent and reliable.

Ion suppression effects were observed for all analytes, being $24.2 \pm 12.3 \%, 24.8 \pm 8.9 \%$, $26.0 \pm 9.5 \%$ the values obtained for TA-d7, TA, and THA, respectively. TA-d7 showed a similar ion suppression effect than THA and TA, and therefore, it is useful to accurately estimate concentrations of both compounds.

\subsubsection{Analysis of plasma samples}

Plasma levels of THA, TA and CORT were measured in all plasma samples. In Figure 5, plasma concentrations of THA and TA are presented together with CORT levels, which were expressed as percentages with respect to the basal concentration of each patient because of the high inter-individual variability in basal concentrations values. All blood samples were collected in the same time period in the morning to reduce the effect of circadian rhythm in CORT measurements (34). Therefore, basal CORT concentrations refer to morning levels.

THA was detected at lower concentrations than TA (Figure 5). While TA was detected up to the last sample collected on day 10 in all patients (concentration range 0.18 to $1.72 \mathrm{ng} / \mathrm{mL}$ on day 10 ), THA was detected in all plasma samples collected up to $24 \mathrm{~h}$ (concentration range $0.09-1.1 \mathrm{ng} / \mathrm{mL})$, and in $88 \%(0.07-0,44 \mathrm{ng} / \mathrm{mL}), 63 \%(0.09-0.23$ $\mathrm{ng} / \mathrm{mL}), 50 \%(0.08-0.18 \mathrm{ng} / \mathrm{mL})$ and $38 \%(0.08-0.18 \mathrm{ng} / \mathrm{mL})$ of the samples collected on days 2, 3, 4 and 10 after administration, respectively. Both compounds presented similar elimination profiles with peak levels after 24-48 h after administration, showing gender differences with plasma TA and THA concentrations higher in males than in females. 
CORT levels presented a decrease after injection, which was greater in males than in females (Figure 5). CORT concentration profiles of each patient are presented in Figure 6. A reversible decrease in plasma CORT concentrations was observed after IA administration of THA in all male patients (Figure 6, subjects 1, 2, 3 and 4) and in two out of the four female patients (Figure 6, subjects 5 and 6). The earliest decrease was seen $1 \mathrm{~h}$ after injection, and the minimum CORT levels appeared in the sample collected $24 \mathrm{~h}$ after administration. For some patients (Figure 6, males 1, 2 and 3 as well as females 5 and 6), the CORT levels remained low during at least $96 \mathrm{~h}$. For patient 4 , CORT began to rise at $72 \mathrm{~h}$ after administration. For males 1 and 2, and female 5, the CORT concentrations were not in basal levels even $240 \mathrm{~h}$ after administration. CORT levels of females 7 and 8 seemed not to be affected by the IA injection (Figure 6). 


\section{Discussion}

Up to our knowledge, no studies on THA urinary metabolism after supervised IA administration of the drug have been described up to now. For this reason, the first objective of the work was to evaluate urinary metabolites of THA, with one of the main focus in the identification of specific metabolites of THA.

Due to the difficulties to obtain ethical approval to perform IA administration in healthy volunteers, the study was performed with patients diagnosed with osteoarthritis of the knee that needed IA administration of THA for therapeutic purposes. The IA dose of THA administered in the clinical trial $(40 \mathrm{mg})$ simulated a therapeutic treatment that could be prescribed to athletes (38). One of the limitations of the use of patients is that their age did not fit with the normal age of athlete populations (Table 1). In addition, most of the patients had concomitant pathologies and were prescribed with other treatments that could affect the parameters that need to be evaluated in the study. All these aspects need to be taken into consideration in the discussion of results and when drawing the conclusions of the study.

Results of the metabolic study in urine demonstrated that THA and potential THA specific metabolites were not detected in urine and only metabolites resulting from the cleavage of the ester bond (TA and TA metabolites) were detected. This indicates the complete cleavage of the ester by plasma esterases from THA to TA. Therefore, there is no likelihood that THA and specific THA metabolites could be used as an unequivocal proof of IA use of THA, which is only marketed for IA use administration.

The main urinary metabolites after IA administration of THA were TA and 6ßOHTA, as described for TA after IM administration of $20 \mathrm{mg}(18,19)$. However, the excretion profiles in urine were different from IM administration. After IA administration, concentrations of all metabolites were very high during the first 4 days after 
administration and decreased over time (up to day 23 post-administration), except for metabolites M4 and M5 that are excreted in low amounts during the first $24 \mathrm{~h}$. After IM administration, more constant values in concentrations were obtained during all the days of the study (up to day 15 post-administration) (19). The slower elimination of TA metabolites after IM administration is probably due to the depot formulation of TA medication administered by IM route (19). In contrast, the medication used for IA administration of THA was an alcoholic solution and no delayed delivery took place.

It is worth to notice that urinary concentrations of TA and its metabolites during the first four days after IA injection were comparatively higher than those obtained after an IM dose of $20 \mathrm{mg}$, described in the previous study (19).

Using the current WADA criterion to distinguish allowed and forbidden administrations of GCs (reporting level of $30 \mathrm{ng} / \mathrm{mL}$ ), a large number of samples collected up to day 4 after IA administration of THA would produce false AAFs in males. The situation would be worse using the discrimination criteria to detect IM administration proposed in a previous study (reporting level of TA at $5 \mathrm{ng} / \mathrm{mL}$ or $6 \beta$ OHTA at $100 \mathrm{ng} / \mathrm{mL}$ ) (19).

In summary, all the results available show that it is not possible to distinguish between IA and IM administration of THA and TA, respectively, using a reporting level for TA or $6 \beta$ OHTA in urine. Taking into account the current WADA rules about the use of GCs (10), a recommendation for athletes could be not to participate at sport competitions during some days after IA administration. This recommendation is reasonable from a medical point of view, since it is recommended to avoid vigorous activity for some days post-injection (6).

Concentrations of TA and some metabolites were greater in male than in female patients. As mentioned above, one of the reasons could be the concomitant use of medications containing diuretic agents in three of the four female patients. However, 
lower concentrations in females were not observed for all metabolites and the differences observed were maintained after correction by SG, suggesting that some gender differences exist in the metabolism and urinary excretion of THA.

Plasma samples were collected in $\mathrm{K}_{2}$ EDTA tubes which may have helped to reduce THA hydrolysis to TA (39). Although there are other esterase inhibitors reported to be more adequate to stabilize THA in plasma (39), blood samples were collected using the containers indicated in the WADA blood sample collection guidelines (35).

Both TA and THA were detected in all post-administration plasma samples from all patients, but THA was detected at lower concentrations. Maximum concentrations were observed at the sample collected at $24 \mathrm{~h}$ after administration. However, the first samples were collected at 1,2 and $24 \mathrm{~h}$, and a previous study showed maximum serum concentrations $8 \mathrm{~h}$ after administration (30). Therefore, the concentrations detected in the samples collected at $24 \mathrm{~h}$ may not be the maximum values achieved. The concentrations of THA in plasma reflect the equilibrium between the delivery of THA to blood stream from the articulation and the elimination of THA via metabolism and excretion. For this reason, THA in plasma is maintained from long time. The possibility to detect THA in plasma opens a window of opportunity to detect IA administration of THA, as it would be an unequivocal proof to demonstrate IA use of THA. It is important to highlight that the additional systemic administration of TA cannot be excluded via this method.

Plasma CORT levels were also monitored to evaluate the effect of the IA administration of THA on the HPA axis. Our study showed that the IA treatment with THA has a profound impact on the HPA axis since CORT levels were largely suppressed in six of 
the eight patients studied. This finding is in agreement with previous studies showing a suppression of HPA axis for one week after IA administration of THA $(29,30)$.

All the participants in the study received additional therapeutic agents for concomitant pathologies. Some of these drugs have shown to modify CORT plasma concentrations in previous clinical assays. Among them, a significant increase in plasma CORT concentrations has been observed following 3-hydroxy-3-methyl-glutaryl-CoA reductase inhibitors like simvastatin or atorvastatin (40) or acetyl salicylic acid (41) treatments, whereas a decrease of CORT concentrations has been described for calcium antagonist treatments with amilodipine (42) or duloxetine (43) and during gabapentin therapy (44). However, all these medications were taken on a regular basis and, their effect on plasma CORT, if any, was already produced in the samples taken before THA injection. Therefore, the reduction of CORT levels observed for most of the participants in the study after IA administration of THA seem to be related to the administration of that drug. In addition, the decrease in plasma CORT observed in our patients was directly proportional to THA and TA plasma concentrations (Figure 5). The HPA axis suppression seemed to be more pronounced in males than in females (Figure 5). These differences are related with plasma THA and TA levels, higher in males than in females. In summary, our study indicates systemic effect after IA administration as it has been already observed in previous studies for THA and for other GCs. In the current WADA prohibited list, IA administration of GCs is allowed without restrictions. However, the existence of systemic effect recommends that the use of GCs by IA administration should be controlled. 


\section{Conclusions}

The assessment of the metabolism of THA in human urine after IA injection showed that only TA and its metabolites were detected in urine. Neither THA nor specific THA metabolites were detected. In plasma, THA and TA were detected in all patients during the first hours after administration and up to day 10 for some of the patients. The detection of THA in plasma would be an unequivocal proof of the IA administration of THA.

A reversible decrease was observed in plasma concentrations of CORT after IA administration of THA in some of the patients, showing that the drug has an impact on the HPA axis, and therefore, systemic effect. The status of the IA administration of THA in the WADA list should be revised.

\section{Acknowledgements}

The authors are grateful for the financial support received from the WADA (research grant 16C11RV) and Consell Català de l'Esport (Generalitat de Catalunya). Support from AGAUR (Generalitat de Catalunya) (2017 SGR 81) is also acknowledged.

\section{Conflict of interest}

The authors wish to disclose that there were no conflicts of interest associated with professional relationships, and that the results of the study are presented clearly, honestly, and without fabrication, falsification, or inappropriate data manipulation. 


\section{REFERENCES}

1. Scherer J, Rainsford KD, Kean CA, Kean WF. Pharmacology of intra-articular triamcinolone. Inflammopharmacol 2014;22(4):201-17.

2. Duclos M. Use and abuse of anabolic steroids and glucocorticoids in sport. Ann Endocrinol 2007;68(4):308-14.

3. Thuyne WV, Delbeke FT. Declared used of medication in sports. Clin J Sport Med 2008;18(2):143-7.

4. Guinot M, Duclos M, Idres $\mathrm{N}$, et al. Value of basal serum cortisol to detect corticosteroid-induced adrenal insufficiency in elite cyclists. Eur J Appl Physiol 2007;99(3):205-16.

5. Dvorak J1, Feddermann N, Grimm K. Glucocorticosteroids in football: use and misuse. Br J Sports Med. 2006;40 Suppl:48-54.

6. Fitch K. Glucocorticoids at the Olympic Games: state-of-the-art review. Br J Sports Med 2016;50(20):1267.

7. Duclos M. Evidence on ergogenic action of glucocorticoids as a doping agent risk. Physician Sportsmed 2010;38(3):121-7.

8. Keller-Wood ME, Dallman MF. Corticosteroid inhibition of ACTH secretion. Endocr Rev 1984;5(1):1-24.

9. Henzen C, Suter A, Lerch E, et al. Suppression and recovery of adrenal response after short-term, high-dose glucocorticoid treatment. The Lancet 2000;355:542-45.

10. The World Anti-Doping Code. The 2019 Prohibited List. International Standard. [World Anti-Doping Agency Website]. Available at: https://www.wadaama.org/sites/default/files/wada_2019_english_prohibited_list.pdf (Accessed January 2019).

11. Deventer K, Mikulcikova P, Van Hoecke H, Van Eenoo P, Delbeke FT. Detection of budesonide in human urine after inhalation by liquid chromatography-mass spectrometry. J Pharm Biomed Anal 2006;42(4):474-479.

12. Pozo OJ, Ventura R, Monfort N, Segura J, Delbeke FT. Evaluation of different scan methods for the urinary detection of corticosteroid metabolites by liquid chromatography tandem mass spectrometry. J Mass Spectrom 2009;44(6):929-44.

13. Panusa A, Regazzoni L, Aldini G, et al. Urinary profile of methylprednisolone acetate metabolites in patients following intra-articular and intramuscular administration. Anal Bioanal Chem 2011;400(1):255-267.

14. Pozo O J, Marcos J, Matabosch X, Ventura R, Segura J. Using complementary mass spectrometric approaches for the determination of methylprednisolone metabolites in human urine. Rapid Commun Mass Spectrom 2012;26(5):541-53. 
15. Matabosch X, Pozo OJ, Pérez-Mañá $C$, et al. Identification of budesonide metabolites in human urine after oral administration. Anal Bioanal Chem 2012;404(2):325-340.

16. Matabosch X, Pozo OJ, Monfort N, et al. Urinary profile of methylprednisolone metabolites after oral and topical administrations. J Steroid Biochem Mol Biol 2013;138:214-21.

17. Matabosch X, Pozo OJ, Pérez-Mañá C, et al. Discrimination of prohibited oral use from authorized inhaled treatment of budesonide in sports. Ther Drug Monit 2013;35(1):118-28.

18. Matabosch X, Pozo OJ, Papaseit E, et al. Detection and characterization of triamcinolone acetonide metabolites in human urine by LC-MS/MS after intramuscular administration. Rapid Commun Mass Spectrom 2014;28(16):1829-39.

19. Matabosch X, Pozo OJ, Pérez-Mañá C, et al. Evaluation of the reporting level to detect triamcinolone acetonide misuse in sports. J Steroid Biochem Mol Biol 2014;145:94-102.

20. Chang CW, Huang TY, Tseng YC, et al. Positive doping results caused by the single-dose local injection of triamcinolone acetonide. Forensic Sci Int 2014;244:1-6.

21. Matabosch X, Pozo OJ, Monfort N, et al. Detection and characterization of betamethasone metabolites in human urine by LC-MS/MS. Drug Test Anal 2015;7:66372 .

22. Simoes SMS, Calçada M, Horta L, de la Torre X. Methylprednisolone detection in urine following local and oral administrations. In. Recent Advances in Doping Analysis (13). Schänzer W, Geyer H, Gotzmann A, Mareck U (Eds). Sportverlag Strauss, Cologne, Germany, 411-414 (2005).

23. Matabosch X, Monfort N, Pozo OJ et al. Urinary profile of betamethasone metabolites after different administration routes. Presented at: 32nd Cologne Workshop on Dope Analysis. Cologne, Germany, 30th March-4th April 2014.

24. Ventura R, Matabosch X, Monfort N, Pozo OJ, Segura J. Urinary profiles of corticosteroids after intra-articular and related administrations. Presented at 33rd Cologne Workshop on Dope Analysis. Cologne, Germany, 1st-6th March 2015.

25. Ventura R, Matabosch X, Segura J. Bioanalytical techniques in discrimination between therapeutic and abusive use of drugs in sport. Bioanalysis 2016;8(9):965-80.

26. Coll S, Matabosch X, Monfort N, et al. Elimination profile of betamethasone and metabolites in urine after different administration routes. Pesented at 22nd International Mass Spectrometry Conference. Florence, Italy, 26th-31th August 2018.

27. Coll S, Matabosch X, Llorente-Onaindia J, et al. Elimination profile of triamcinolone hexacetonide and its metabolites in human urine and plasma after a single 
intra-articular administration. Presented at 37th Cologne Workshop on Dope Analysis. Cologne, Germany, 17th-21th February 2019.

28. Gless KH, Klee HR, Vecsei P, Weber M, Haack D, Lichtwald K. Plasma concentration and systemic effect of betamethasone after intra-articular injection. Dtscg Med Wochenschr 1981;106(22):704-07.

29. Derendorf H, Mollmann H, Gruner A, Haack D, Gyselby G. Pharmacokinetics and pharmacodynamics of glucocorticoid suspensions after intra-articular adminsitration. Clin Pharmacol Ther 1986;39(3):313-17.

30. Weitoft T, Rönnblom L. Glucocorticoid resorption and influence on the hypothalamic-pituitary-adrenal axis after intra-articular treatment of the knee in resting and mobile patients. Ann Rheum Dis 2006;65(7):955-57.

31. Habib GS. Systemic effects of intra-articular corticosteroids. Clin Rheumatol 2009;28(7):749-756.

32. Catalano LW, Glikel SZ, Barron OA, Harrison R, Marshall A, Purcelli-Lafer M. Effect of local corticosteroid injection of the hand and wrist on blood glucose in patients with diabetes mellitus. Orthopedics 2012;35812):1754-1758.

33. Berkoff DJ, Miller LE, Block JE. Clinical utility of ultrasound guidance for intraarticular knee injectons. Clin Intervention Aging 2012;7:89-95.

34. Guignard MM, Pesquies PC, Serrurier BD, Merino DB, Reinberg AE. Circadian rhythms in plasma levels of cortisol, dehydroepiandrosterone, $\Delta 4$-adrostenedione, testosterone and dihydrotestosterone of healthy young men. Acta Endoc. 1980;94:53645 .

35. WADA ISTI - Blood Sample Collection Guidelines [World Anti-Doping Agency Website]. Available at: https://www.wadaama.org/sites/default/files/resources/files/guidelines_blood_sample_collection_v5_sept _2016.pdf (Accessed September 2018).

36. WADA Technical Document - TD2018DL [World Anti-Doping Agency Website]. Available at: https://www.wadaama.org/sites/default/files/resources/files/td2018dl_v1_en.pdf (Accessed September 2018).

37. Levine L, Fahy JP. Evaluation of urinary lead determinations. J. Ind. Hyg. Toxicol. 1996;20:379-391.

38. Centeno LM, Moore ME. Preferred intraarticular corticosteroids and associated practice: a survey of members of the American college of rheumatology. Arthritis Care Res 1994;7(3):151-5.

39. Sun W, Ho S, Fang XR, O'Shea T, Liu H. Simultaneous determination of triamcinolone hexacetonide and triamcinolone acetonide in rabbit plasma using a highly 
sensitive and selective UPLC-MS/MS method. $J$ Pharm Biomed Anal. 2018;153(10):267-73.

40. Sahebkar A, Rathouska J, Simental-Mendía LE, Nachtigal P. Statin therapy and plasma cortisol concentrations: A systematic review and meta-analysis of randomized placebo-controlled trials. Pharmacol Res 2016;103:17-25.

41. Hervey GR, Hutchinson J. Anabolic effects of methandienone in men undergoing athletic training. Lancet 1976;2(7988):699-702.

42. Beer NA, Jakubowicz DJ, Beer RM, Nestler JE. The calcium channel blocker amlodipine raises serum dehydroepiandrosterone sulfate and androstenedione, but lowers serum cortisol, in insulin-resistant obese and hypertensive men. $J$ Clin Endocrinol Metab 1993;76(6):1464-9.

43. Beck J, Bruni N, Brand S, Holsboer-Trachsler E. Repeated cortisol awakening response as predictor of antidepressant treatment outcome with duloxetine. Neuropsychobiology 2015;71(2):97-102.

44. Karbić VO, Škoda M, Antončić D, Krištofić I, Komar D, Trobonjača Z. Gabapentin-induced changes of plasma cortisol level and immune status in hysterectomized women. Int Immunopharmacol 2014;23(2):530-6. 


\section{Tables}

Table 1. Characteristics of the patients participating in the study.

\begin{tabular}{lccccc}
\hline Gender & $\mathrm{n}$ & age (years) & height $(\mathrm{cm})$ & weight $(\mathrm{kg})$ & BMI $\left(\mathrm{kg} / \mathrm{m}^{2}\right)$ \\
\hline Male & 4 & $68 \pm 5$ & $175 \pm 4$ & $87 \pm 9$ & $28 \pm 2$ \\
Female & 4 & $70 \pm 5$ & $160 \pm 7$ & $66 \pm 2$ & $26 \pm 3$ \\
\hline
\end{tabular}

Table 2. Conditions of analysis of THA and metabolites in human urine by LC-MS/MS. Retention time (RT), monoisotopic mass (MM), precursor and product ions (m/z), and collision energy (CE). The first ion transition indicated for each metabolite was used for quantification purposes. Cone voltage was set to $15 \mathrm{~V}$ for all ion transitions except for ion transitions of THA, which CV was set to $25 \mathrm{~V}$.

\begin{tabular}{|c|c|c|c|c|c|}
\hline Metabolite & RT (min) & MM (Da) & Precursor ion & Product ion(s) & CE $(e V)$ \\
\hline M2 & 5.0 & 450 & 451 & $\begin{array}{l}387 \\
413 \\
227\end{array}$ & $\begin{array}{l}12 \\
13 \\
30\end{array}$ \\
\hline Triamcinolone $(\mathrm{T})$ & 6.2 & 394 & 395 & $\begin{array}{l}375 \\
357 \\
147\end{array}$ & $\begin{array}{c}8 \\
13 \\
30\end{array}$ \\
\hline 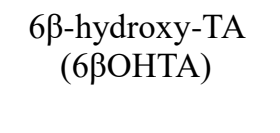 & 7.8 & 450 & 451 & $\begin{array}{l}387 \\
413 \\
227\end{array}$ & $\begin{array}{l}12 \\
13 \\
30\end{array}$ \\
\hline M4 & 12.2 & 452 & 453 & $\begin{array}{l}415 \\
435 \\
357\end{array}$ & $\begin{array}{l}12 \\
20 \\
20\end{array}$ \\
\hline M3 & 14.7 & 432 & 433 & $\begin{array}{l}355 \\
337 \\
121\end{array}$ & $\begin{array}{l}15 \\
20 \\
50\end{array}$ \\
\hline TA & 17.2 & 434 & 435 & $\begin{array}{l}397 \\
339 \\
147\end{array}$ & $\begin{array}{l}13 \\
20 \\
35\end{array}$ \\
\hline M5 & 18.1 & 434 & 435 & $\begin{array}{l}121 \\
357 \\
147\end{array}$ & $\begin{array}{l}50 \\
20 \\
36\end{array}$ \\
\hline THA & 25.2 & 532 & 533 & $\begin{array}{l}513 \\
415 \\
495\end{array}$ & $\begin{array}{c}5 \\
25 \\
25\end{array}$ \\
\hline 16 $\alpha$ OHPRED-d $5^{\mathrm{a}}$ & 6.9 & 381 & 382 & 229 & 26 \\
\hline TA-d $7^{\mathrm{b}}$ & 17.0 & 441 & 442 & $\begin{array}{l}404 \\
340\end{array}$ & $\begin{array}{l}13 \\
20\end{array}$ \\
\hline
\end{tabular}

a) $16 \alpha \mathrm{OHPRED}-\mathrm{d} 5$ was used as ISTD for $6 \beta \mathrm{OHTA}$, T and M-2.

b) TA-d7 was used as ISTD for THA, TA, M-3, M-4 and M-5. 
Table 3. Conditions of analysis of TA, THA and CORT in human plasma. Retention time (RT), monoisotopic mass $(\mathrm{MM})$, precursor and product ions $(\mathrm{m} / \mathrm{z})$, and collision energy (CE). The first ion transition indicated for each metabolite was used for quantification purposes. Cone voltage was set to $20 \mathrm{~V}$ for all TA and THA ion transitions, and to $25 \mathrm{~V}$ for all CORT ion transitions.

\begin{tabular}{cccccc}
\hline Metabolite & RT (min) & MM (Da) & Precursor ion & Product ion(s) & CE (eV) \\
\hline CORT & 6.1 & 362 & 363 & 121 & 25 \\
& & & & 97 & 25 \\
TA & 9.2 & 434 & 435 & 415 & 8 \\
& & & & 397 & 15 \\
& & & & 339 & 20 \\
THA & 16.5 & 532 & 533 & 415 & 15 \\
& & & & 533 & 10 \\
& & & & 495 & 15 \\
CORT-d4 ${ }^{\mathrm{a}}$ & 6.1 & 366 & 367 & 121 & 25 \\
TA-d $7^{\mathrm{b}}$ & 9.0 & 441 & & & \\
\hline
\end{tabular}

a) CORT-d4 was used as ISTD for CORT.

b) TA-d7 was used as ISTD for THA and TA. 
Table 4. Concentrations $(\mathrm{ng} / \mathrm{mL})$ of TA and $6 \beta O H T A$ after IA administration of THA.

\begin{tabular}{|c|c|c|c|c|c|c|c|c|c|c|c|}
\hline \multirow[b]{2}{*}{ Compound } & \multirow[b]{2}{*}{ Time (h) } & \multicolumn{5}{|c|}{ Males (ng/mL) } & \multicolumn{5}{|c|}{ Females (ng/mL) } \\
\hline & & Min & IQ1 & median & IQ3 & $\max$ & $\min$ & IQ1 & median & IQ3 & $\max$ \\
\hline \multirow[t]{7}{*}{ TA } & 12 & 4.7 & 6.4 & 28.2 & 56.1 & 59.9 & 3.3 & 4.2 & 8.6 & 28.7 & 34.8 \\
\hline & 24 & 14.5 & 17.7 & 28.9 & 45.0 & 49.8 & 4.1 & 7.2 & 16.8 & 23. & 25.5 \\
\hline & 48 & 21.4 & 26.5 & 42.1 & 59.7 & 65.5 & 5.0 & 6.3 & 17.5 & 26.5 & 27.0 \\
\hline & 72 & 12.2 & 15.9 & 28.4 & 46.5 & 52.1 & 1.0 & 3.4 & 13.8 & 24.4 & 26.8 \\
\hline & 96 & 7.8 & 10.6 & 25.7 & 33.3 & 33.6 & 2.7 & 4.3 & 9.7 & 18.3 & 21.0 \\
\hline & $120-168$ & 3.9 & 5.8 & 11.6 & 17.6 & 18.0 & 1.1 & 2.9 & 9.2 & 11.7 & 26.0 \\
\hline & $192-552$ & 0.0 & 0.9 & 2.2 & 4.4 & 13.9 & 0.4 & 1.4 & 2.5 & 5.0 & 13.1 \\
\hline \multirow[t]{7}{*}{ 6ßОНТА } & 12 & 92.2 & 94.6 & 157.5 & 435.3 & 509.4 & 52.0 & 71.8 & 174.3 & 387.8 & 444.5 \\
\hline & 24 & 288.4 & 294.5 & 325.6 & 440.5 & 474.5 & 73.2 & 122.1 & 277.7 & 311.3 & 319.5 \\
\hline & 48 & 287.7 & 311.1 & 485.2 & 609.0 & 615.7 & 118.4 & 146.4 & 267.8 & 342.7 & 355.2 \\
\hline & 72 & 220.6 & 242.2 & 361.5 & 432.2 & 437.5 & 28.9 & 74.1 & 239.9 & 401.7 & 445.5 \\
\hline & 96 & 168.8 & 170.1 & 264.1 & 444.2 & 474.2 & 60.0 & 71.0 & 134.4 & 281.1 & 319.9 \\
\hline & $120-168$ & 87.8 & 104.5 & 117.6 & 247.0 & 283.3 & 18.6 & 55.2 & 158.8 & 260.0 & 465.2 \\
\hline & $192-552$ & 7.4 & 17.4 & 35.0 & 60.6 & 238.5 & 14.2 & 29.8 & 63.0 & 102.0 & 330.2 \\
\hline
\end{tabular}




\section{Figure legends}

Figure 1. Chemical structures of the compounds under study: A, THA; B, TA and its metabolites detected in previous studies $(18,19)$.

Figure 2. Collision induced dissociation mass spectra of $[\mathrm{M}+\mathrm{H}]^{+}(\mathrm{A})$ and $[\mathrm{M}+\mathrm{HCOO}]^{-}$ (B) of THA at different collision energies (CE). Cone voltages: A, $25 \mathrm{~V}$; B, $15 \mathrm{~V}$.

Figure 3. Concentrations of TA and $6 \beta \mathrm{OHTA}$ at different times after IA administration of THA. Dotted line corresponds to the reporting level of $30 \mathrm{ng} / \mathrm{mL}$ for GCs.

Figure 4. Amounts of $\mathrm{T}$ and 4 additional TA metabolites at different times after IA administration of THA. For T, concentrations (ng/mL) are shown. For M2 to M5, boxplots were constructed using relative signal to the ISTD.

Figure 5. Plasma levels of TA, THA (concentrations, box plots) and CORT (percentage, mean+SD) at different times after IA administration of THA.

Figure 6. Individual plasma CORT concentrations before and after IA administration of THA. 